\title{
A Rapid Method for Titration of Recombinant Baculovirus Stock Based on Bicistronic Expression of Green Fluorescent Protein (GFP) Reporter
}

\author{
Deepika Bisht ${ }^{1}$, Supriya Bhattacharya ${ }^{1}$, Lekshmi S. Rajan ${ }^{1}$, Mashidur Rana ${ }^{2}$, \\ Ram Bachan ${ }^{2}$, T. Rama ${ }^{2}$, Mohini Saini ${ }^{2}$, and Praveen K. Gupta ${ }^{*}$ \\ ${ }^{1}$ Division of Veterinary Biotechnology, Indian Veterinary Research Institute, Izatnagar- \\ 243122, Uttar Pradesh, India \\ ${ }^{2}$ Division of Biochemistry, Indian Veterinary Research Institute, Izatnagar-243122, Uttar \\ Pradesh, India, India \\ *Corresponding author
}

\section{A B S T R A C T}

Keywords

Baculovirus, FLAG, Sf21 insect cells, IRES, GFP, PCR

Article Info

Accepted:

10 November 2018

Available Online:

10 December 2018
To determine the titer of baculovirus stock accurately based on green fluorescent protein (GFP) reporter, a recombinant baculovirus encoding FLAG-tagged protein and GFP reporter as bicistronic messenger was constructed. The bicistronic messenger RNA was transcribed using polyhedrin promoter and translated in infected Sf21 insect cells. The GFP reporter gene was expressed incap independent manner utilizing internal ribosome entry site (IRES) which helped in titration of baculovirus using end point titration method. The result of this study indicated that recombinant baculovirus expressing GFP reporter as bicistronic messenger facilitated in titration of baculovirus in stock in rapid and accurate manner.

\section{Introduction}

The baculovirus expression vector system (BEVS) is one of the most powerful tools for the production of recombinant proteins on both laboratory and industrial scales for various applications such as production of vaccines and pharmaceutical proteins $(\mathrm{O}$ Reilly et al., 1992; Meghrous et al., 2009; Cox, 2012). The robustness and efficiency of the protein production using BEVS depends on optimization of a series of critical process parameters, including the ratio of input baculovirus to number of Sf21 insect cells in the culture, also referred as the multiplicity of infection (MOI). In accurate estimation of baculovirus titers has a profound impact on the process performance and batch consistency. Under estimated baculovirus titers result in higher MOIs than expected leading to a severe metabolic burden effect and causing low protein productivity. Similarly, overestimated titers result in low production levels due to non-synchronous 
infection (Roldao et al., 2008).Therefore, accurate measurement of virus titer before protein production is much essential.

Conventional baculovirus titration methods include plaque assay and end point dilution based on cell viability (Lynn, 1992; Mena et al., 2003; Janakiraman et al., 2006). All these methods rely on cellular morphological changes caused by infection such as the decrease of cell viability and the increase of cell size. The extent of those phenomena is dependent on the virus dose and thus can be correlated with viral titres. The accuracy of the titer determination is greatly dependent on the operator's skill and experience which limit its widespread applications.

In this study, we report a rapid and reproducible baculovirus titer method based on the detection of green fluorescent protein (GFP) reporter gene expression in baculovirus infected insect cells for accurate estimation of baculovirus titer in stock. The GFP reporter gene expression was detected using fluorescent microscope and used to determine virus titer. The proposed GFP reporter based baculovirus titration method is rapid, simpleand accurate when compared with gp64 gene based PCR assay.

\section{Materials and Methods}

Production of recombinant baculovirus encoding FLAG-tagged protein and GFP as bicistronic messenger

The gene encoding FLAG-tagged protein was amplified using PCR and cloned into pSMPUW-IRES-GFP expression vector(Cell Biolabs) between EcoRV and EcoRI restriction sites. The recombinant pSMPUWIRES-GFP plasmid was digested with EcoRV and SalI restriction endonucleases and the EcoRV-SalI DNA fragment containing FLAG-tagged protein, IRES and GFP was subcloned into pFastBac-1 baculovirus donor plasmid (Invitrogen) between StuI-XhoI restriction sites. The recombinant pFastBac-1 donor plasmid used to construct recombinant baculovirus using Bac-to-bac system (Invitrogen) following manufacturer's instructions. The recombinant baculovirus encoded FLAG-tagged protein and GFP as bicistronic messenger under the control of polyhedrin promoter (Fig. 1A).

\section{Detection of FLAG-tagged protein and GFP in baculovirus infected Sf 21 cells}

The expressed GFP and FLAG-tagged proteins in baculovirus infected Sf21 cells were detected using fluorescent microscopy and indirect fluorescent antibody test (IFAT), respectively. For detection of GFP in infected Sf 21 cells, the Sf21 cells were infected with recombinant baculovirus and expressed GFP in cells were analysed on 2 days post-infection under fluorescent microscope using GFP filter. For detection of FLAG-tagged protein, the Sf21 cells-infected with recombinant baculovirus at 2 days post-infection were fixed with Methanol:Acetone (1:1) for $30 \mathrm{~min}$ at $20^{\circ} \mathrm{C}$. The Methanol:Acetone fixing denatured the GFP which resulted in no green fluorescence in fixed cells. The FLAG-tagged protein in Sf21 cells were probed with rabbit anti-FLAG polyclonal antibody diluted in PBS-T (PBS containing 0.1\% Tween-20). After washing with PBS-T, the bound antibodies were detected with anti-rabbit antibody labelled with FITC diluted in PBS-T. The stained cells were examined under fluorescent microscope using FITC filter after counter stained with DAPI nuclear stain. The non-infected healthy Sf21 cells were included in the study as control.

\section{End point titration of baculovirus using GFPas reporter}

For determination of baculovirus titer baculovirus stock prepared from infected Sf21 cell culture supernatant was diluted 10-fold 
from $10^{-1}$ to $10^{-10}$ and used as inoculum. Approximately 50,000 Sf21 cells in $100 \mu 1$ SFM per well were seeded in 96-well plate and incubated at $27^{\circ} \mathrm{C}$ to allow attachment of cells. After $2 \mathrm{~h}$ of incubation, the attached Sf21 cells were infected with $100 \mu$ of different dilutions of baculovirus in quadruplets and incubated further for 7 days. The plate was examined under the fluorescent microscope for detection of GFP in each well indicating presence of baculovirus. The baculovirus titer in stock was calculated using the method of Reed and Muench (1938).

\section{Determination of baculovirus titer using PCR}

For determination of baculovirus titer using PCR, $100 \mu \mathrm{l}$ of the 10 -fold diluted baculovirus from $10^{-1}$ to $10^{-10}$ was used as inoculum to infect Sf21 cells. After 7 days post-infection, the total DNA was isolated from the infected cells and used as template in PCR to detect baculovirus gp64 gene. Briefly, total DNA was isolated from the infected cells in $200 \mu \mathrm{l}$ volume using QIAamp DNA mini kit (Qiagen) following manufacturer's instructions and used as template in PCR. The PCR reaction contained $10 \mu \mathrm{l}$ of isolated total DNA, $50 \mu \mathrm{M}$ each of forward (5'-CACCACACGTGCAAC AAATC-3') and reverse (5'-GAATCATACT CACGCCGTCT C-3') primers, $200 \mu \mathrm{M}$ each dNTPs, 2.5 U Taq DNA polymerase (New England Biolabs) in $1 \mathrm{X}$ reaction buffer in 50 $\mu \mathrm{l}$ reaction volume. The cycling condition was $94^{\circ} \mathrm{C}$ for 3 min initial denaturation, and then 30 cycles of $94^{\circ} \mathrm{C}$ for $1 \mathrm{~min}, 55^{\circ} \mathrm{C}$ for $30 \mathrm{sec}$, and $72^{\circ} \mathrm{C}$ for $1 \mathrm{~min}$. The reaction mixture was then incubated at $72^{\circ} \mathrm{C}$ for $10 \mathrm{~min}$ and stored at $4^{\circ} \mathrm{C}$.An aliquot of $10 \mu \mathrm{l}$ amplified PCR reaction was run in $1 \%$ agarose gel along with 100 bp DNA ladder and gp64 specific 175 bp PCR product was analysed. A negative control reaction containing distilled water as template was included in the assay. The baculovirus titer was calculated as reciprocal of the dilution of baculovirus stock as inoculum which yielded 175 bp PCR product.

\section{Results and Discussion}

Production of recombinant baculovirus expressing FLAG-tagged protein and GFP using bicistronic messengerRNA

As per the manufacturer's protocol, the Sf 21 cells were transfected with recombinant bacmid encoding FLAG-tagged and GFP as bicistronic messenger RNA to produce recombinant baculovirus expressing FLAGtagged and GFP proteins. The transfected Sf 21 cells were incubated at $27^{\circ} \mathrm{C}$ for 5 days and cell culture supernatant was harvested as $\mathrm{P0}$ stock. The P0 stock was used as inoculum to infect healthy Sf21 cells at 0.01 multiplicity of infection (MOI) to prepare $\mathrm{P} 1$ and $\mathrm{P} 2$ baculovirus stocks. The baculovirus titer in $\mathrm{P} 2$ stock was determined after infecting Sf21 which showed green fluorescence cells at 2 days post-infection (Fig. 1B). This indicated that GFP was expressed in infected Sf21 cells utilizing IRES sequences on bicistronic messenger in a cap-independent manner. To confirm the expression of FLAG-tagged protein, the infected $S f 21$ cells were fixed with Methanol:Acetone mixture and probed with anti-FLAG antibody. The green fluorescence in fixed cells indicated that the FLAG-tagged protein was expressed from bicistronic messenger RNA (Fig. 2). There was no fluorescence observed in control non-infected Sf 21 cells. This confirmed that FLAG-tagged protein was expressed in infected Sf 21 cells from bicistronic messenger RNA transcribed using baculovirus polyhedrin promoter in cap dependent manner.

\section{Determination of baculovirus titer using GFP as reporter}

On 7 days post infection, all four wells of Sf 21 cells infected with dilutions from $10^{-1}$ to $10^{-5}$ of 
recombinant baculovirus demonstrated GFP expression. Three out of 4 wells of $10^{-6}$ dilution also showed GFP. There was no GFP expression in $\mathrm{Sf} 21$ cells infected with dilutions from $10^{-7}$ to $10^{-10}$ of recombinant baculovirus. This indicated that the titer of recombinant baculovirus stock was $10^{-7.3} \mathrm{TCID}_{50} / \mathrm{ml}$ (Table $1)$.

\section{Determination of baculovirus titer using gp64 PCR}

When total DNA isolated from SF21 cells infected with $100 \mu$ l of different dilutions of recombinant baculovirus was used in PCR using gp64 specific primers, there was amplification of $175 \mathrm{bp}$ PCR product in total DNA isolated from Sf21 cells infected with dilutions from $10^{-1}$ to $10^{-6}$ of recombinant baculovirus. There was no amplification in total DNA isolated from Sf21 cells infected with dilutions from $10^{-7}$ to $10^{-10}$ of recombinant baculovirus as well as in non-template control (Figure 3). This indicated that the titer of recombinant baculovirus stock was $10^{-6} / 100 \mu 1$ or $10^{-7} / \mathrm{ml}$ stock.

Table.1 Titration of baculovirus stock based on GFP as reporter using Reed and Muench (1938) method

\begin{tabular}{|c|c|c|c|c|c|c|}
\hline Dilution & $\begin{array}{c}\text { No. of } \\
\text { Positive } \\
\text { wells }\end{array}$ & $\begin{array}{c}\text { No. of } \\
\text { negative } \\
\text { wells }\end{array}$ & $\begin{array}{c}\text { Cumulative } \\
\text { positive } \\
\text { wells }\end{array}$ & $\begin{array}{c}\text { Cumulative } \\
\text { Negative } \\
\text { wells }\end{array}$ & $\begin{array}{c}\text { Ratio } \\
\text { (Positive/total) }\end{array}$ & Ratio\% \\
\hline $\mathbf{1 0}^{-1}$ & 4 & 0 & 23 & 0 & $23 / 23$ & 100 \\
\hline $\mathbf{1 0}^{-2}$ & 4 & 0 & 19 & 0 & $19 / 19$ & 100 \\
\hline $\mathbf{1 0}^{-3}$ & 4 & 0 & 15 & 0 & $15 / 15$ & 100 \\
\hline $\mathbf{1 0}^{-4}$ & 4 & 0 & 11 & 0 & $11 / 11$ & 100 \\
\hline $\mathbf{1 0}^{-5}$ & 4 & 0 & 7 & 0 & $7 / 7$ & 100 \\
\hline $\mathbf{1 0}^{-6}$ & 3 & 1 & 3 & 1 & $3 / 4$ & 75 \\
\hline $\mathbf{1 0}^{-7}$ & 0 & 4 & 0 & 5 & $0 / 5$ & 0 \\
\hline $\mathbf{1 0}^{-8}$ & 0 & 4 & 0 & 9 & $0 / 9$ & 0 \\
\hline $\mathbf{1 0}^{-9}$ & 0 & 4 & 0 & 13 & $0 / 13$ & 0 \\
\hline $\mathbf{1 0}^{-\mathbf{1 0}}$ & 0 & 4 & 0 & 17 & $0 / 17$ & 0 \\
\hline
\end{tabular}

Proportionate distance $=(75-50) /(75-0)=25 / 75=0.3$

Thus titre $=10^{-(6+0.3)}=10^{-6.3} / 100 \mu 1$

or $10^{-7.3} / \mathrm{ml}$ stock 
Fig.1 Expression of green fluorescent protein (GFP) reporter gene using bicestronic messenger in baculovirus infected Sf21 insect cells

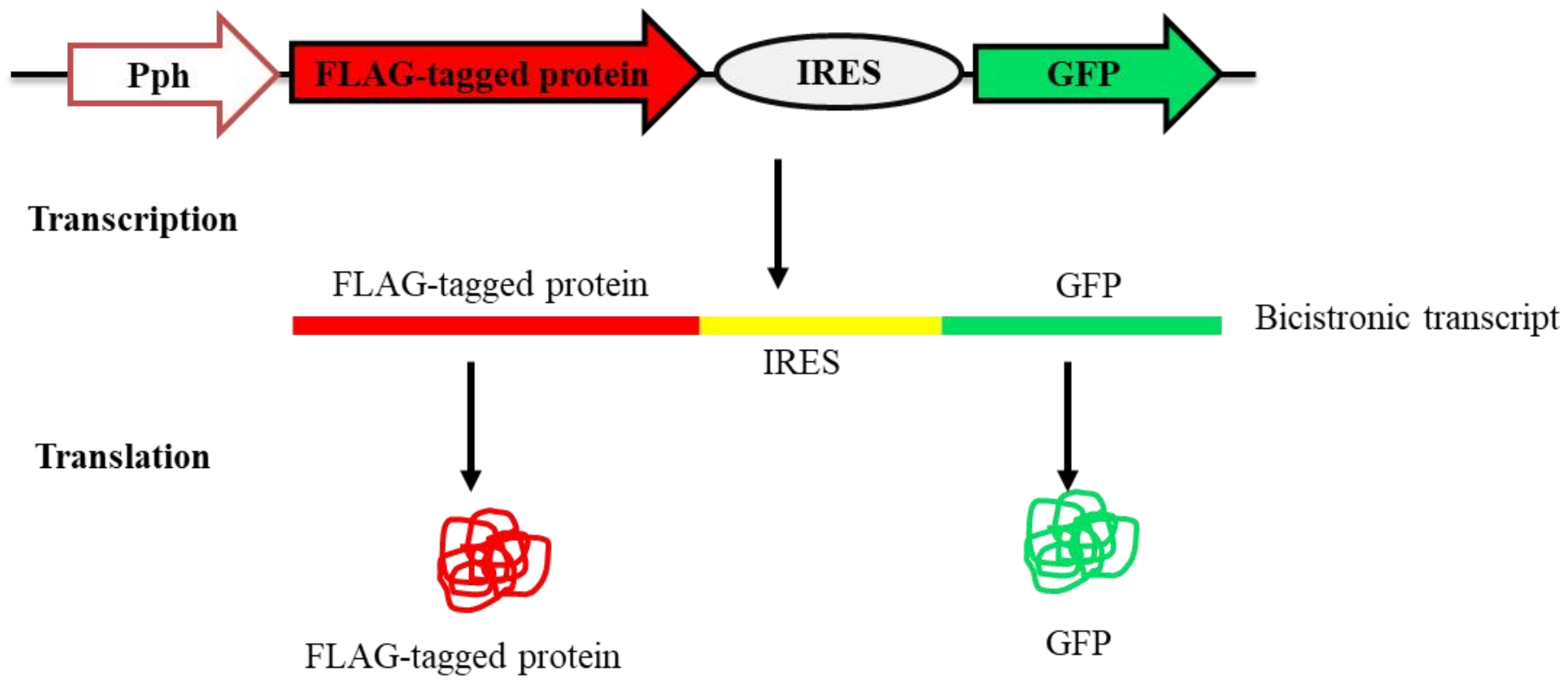

A

A.

Bright field

GFP

Merged
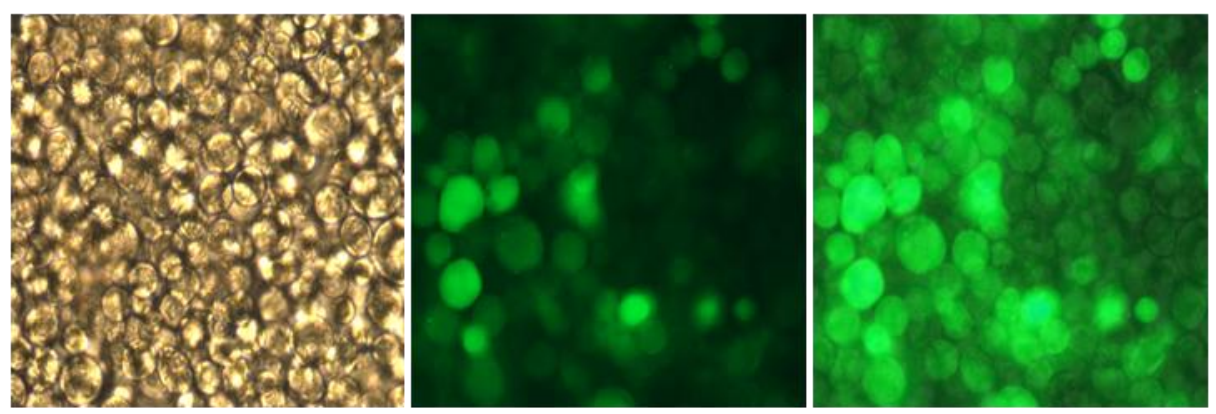

B

B. Schematic representation of bicistronic expression cassette under the control of baculovirus polyhedrin promoter (Pph). The bicistronic messenger RNA encoding FLAG-tagged and green fluorescent protein

C. The recombinant baculovirus encoding FLAG-tagged and green fluorescent protein (GFP) proteins was used to infect Sf21 insect cells. At 2 days post infection, the GFP as reporter was detected in infected cells. 
Fig.2 Indirect fluorescent antibody test (IFAT) of baculovirus infected Sf21 cells for detection of FLAG-tagged protein

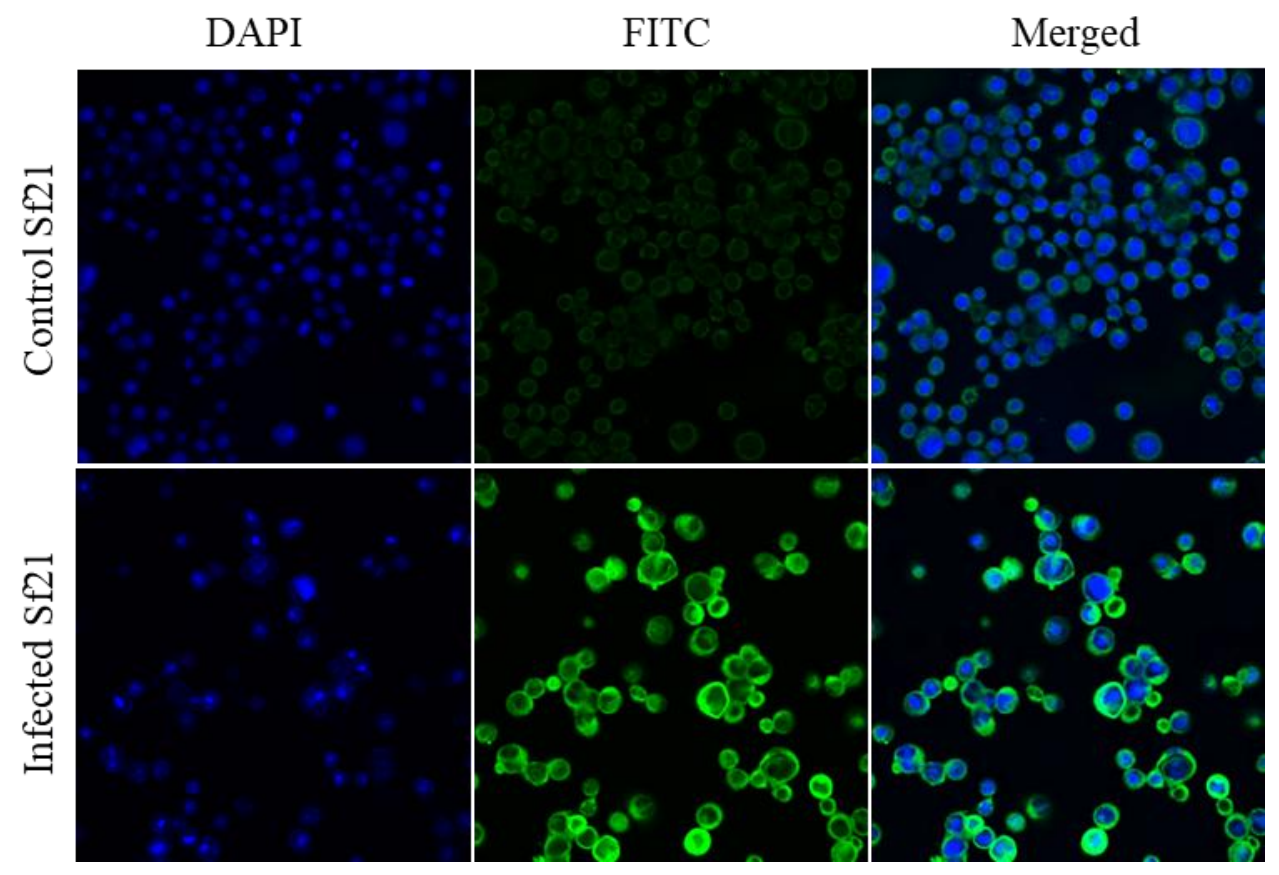

Baculovirus infected Sf21 cells were probed with rabbit anti-FLAG polyclonal antibody and bound antibodies were detected using anti-rabbit antibody conjugated with FITC

Fig.3 Detection of baculovirus in total DNA isolated from infected Sf21 cells using PCR. Total DNA was isolated from Sf21 cell lysate infected with different dilutions of baculovirus and used in PCR to detect gp64 gene. M: 100 bp plus DNA ladder

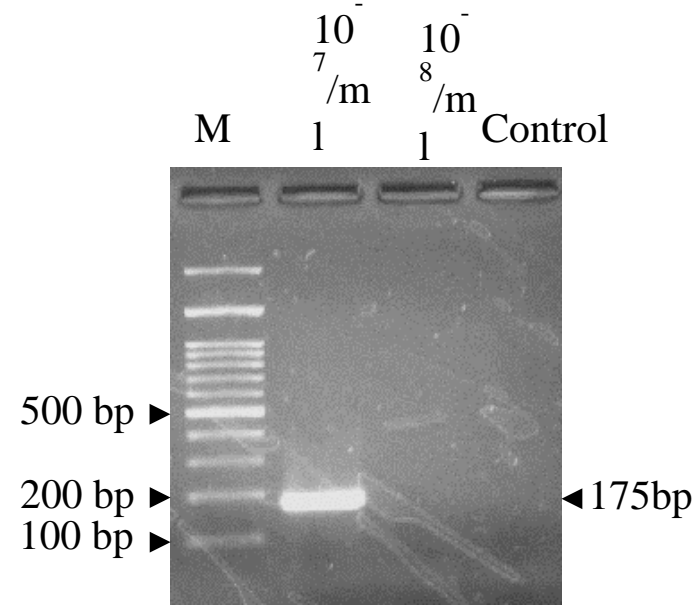


In biological research, co-expression of two or more genes, one being selectable marker and others as gene of interest is often desirable. The ability of IRES sequences to initiate cap independent translation instead of using two or more promoters facilitates expression of more than one polypeptide from single bicistronic/polycistronic expression vector in single host cell (Roberts et al., 1998; Han and Zhang, 2006). This becomes beneficial especially for expressing multisubunit protein where all the subunits are translated at same time in single cell and assemble in its functional quaternary structure (Berger et al., 2004). For past many years, bicistronic expression vectors with IRES sequences have been employed for various experimental settings from mammalian culture cells (Kuo et al., 2016) to baculoviral expression system (Chen et al., 2005) and transgenic animals (Trichas et al.,2008).

In present study, we used baculovirus expression vector system (BEVS) to demonstrate that bicistronic expression of GFP reporter gene utilizing IRES sequence can facilitate accurate titration of baculovirus in stocks. The advantage of BEVS is better yield of desired product as compared to its mammalian counterpart. The yield of desired protein depends on various critical factors such asMOI of infection, time of harvest and temperature (Metz et al., 2011) etc. Therefore before use, titration of baculoviral stock is important to avoid batch to batch variation and maintenance of repeatability of a protocol. Now a days, various methods have been developed for titration, including immunostaining with monoclonal antibodies against baculovirus proteins major coat protein VP39 (Wang et al., 2013)and surface glycoprotein 64 (gp64) (Mulvaniaet al., 2004), direct staining of virus particles using SYBR Green I followed by flow cytometry (FCM) (Shen et al., 2002), cell viability assay with Alamar Blue (Pouliquen et al., 2006), measurement of cell-diameter change of infected cells using a cell counter (Janakiraman et al., 2006) and titration assay based on viable cell side scatter (Qi et al., 2015). Nevertheless, these methods have several drawbacks which limit their widespread applications. For example, immunological staining methods need antibodies of high quality which are very expensive or are not commercially available. AlamarBlue monitors the cell growth cessation induced by baculovirus infection, but the procedure is complex and the fluorescence dye is also expensive. Using cell size method, the diameter of infected cells only increased by $10-40 \%$, leading to limited accuracy and sensitivity when the alteration is not obvious. This method further becomes of limited use when working with Sf21 cells which unlike Sf9 cells are normally irregular in size. Viable cell side scatter based titration assay is comparatively faster method but it only measures granulation in the infected cells which cannot differentiate between infection of wild-type or recombinant baculovirus.

In present study, we expressed FLAG-tagged protein under control of polyhedrin promoter in conjunction with cap independent expression of GFP reporter gene downstream to $616 \mathrm{bp}$ long IRES sequence. Expression of GFP helped in confirming production of recombinant baculovirus and tracing the infected cells. The GFP expression was utilized for titration of recombinant baculovirus as a simplified method compared to the conventional way of neutral red stain mixed overlay technique of viral plaques for baculoviral titration. The estimated titer using GFP based method was further confirmed by PCR amplification of gp64 gene amplification of baculovirus. PCR based titration validated the GFP based titration method as comparable baculovirus titer was estimated using both techniques. 
This system of polycistronic expression in BEVs and reporter gene based titration methods thus can be used in future for development of multisubunit proteins and virus like particles comprising more than one protein. It helped in tracing production of recombinant baculovirus for using appropriate MOI for amplification of baculovirus or protein expression work and optimising the time to harvest supernatant for maximum product yield. The IRES based reporter gene expression also avoids incorporation of unwanted reporter protein into the desired protein thus avoiding change in the conformation or subsequent loss of function of the protein. The titration based on bicistronic expression of GFP utilizing IRES is rapid technique for titration, which do not require any labelling or washing steps of antibody and fluorescence dye and simultaneously can differentiate between recombinant and wild type baculovirus. The result of this study indicated that recombinant baculovirus expressing GFP reporter as bicistronic messenger facilitated in titration of baculovirus in stock in rapid, simple and accurate manner.

\section{Acknowledgements}

The authors thank to the Director, Indian Veterinary Research Institute (IVRI) for providing necessary facilities to carry out the work. This work was supported by grant BT/PR16025/NER/95/52/2015 from the Department of Biotechnology, Government of India.

\section{Conflict of Interest}

All authors declare no conflict of interest.

\section{References}

Berger, I., Fitzgerald, J., Richmond, T.J. 2004. Baculovirus expression system for heterologous multiprotein complexes. Nat. Biotechnol., 22:15831587.

Chen, Y.-J., Chen, W.-S., Wu, T.-Y. 2005. Development of a bi-cistronic baculovirus expression vector by the Rhopalosiphum padi virus 5' internal ribosome entry site. Biochem. Biophys. Res. Comm., 335: 616-623.

Cox, M.M.2012. Recombinant protein vaccines produced in insect cells, Vaccine, 30: 1759-1766.

Han, F., Zhang, X. (2006). Internal initiation of mRNA translation in insect cell mediated by an internal ribosome entry site (IRES) from shrimp white spot syndrome virus (WSSV) Biochem. Biophys. Res. Comm.,344:893-899.

Janakiraman, V., Forrest, W.F., Chow, B., Seshagiri, S. 2006. A rapid method for estimation of baculovirus titer based on viable cell size.J. Virol. Methods, 132: 48-58.

Kuo, S.-C., Teng, C.-Y., Ho, Y.-J., Chen, Y.J., Wu, T.-Y. 2016. Using Bicistronic Baculovirus Expression Vector System to Screen the Compounds That Interfere with the Infection of Chikungunya Virus, Chikungunya Virus: Methods and Protocols, Methods in Molecular Biology 1426: 263-272

Lynn, D.E. 1992. Improved efficiency in determining the titer of the Autographa californica baculovirus nonoccluded virus. Biotechniques, 13: 282-285.

Meghrous. J., Mahmoud, W., Jacob, D., Chubet, R., Cox, M., Kamen, A.A. 2009. Development of a simple and high-yielding fed-batch process for the production of influenza vaccines. Vaccine, 28: 309-316.

Mena, J., Ramirez, O.T., Palomares, L. 2003. Titration of non-occluded baculovirus using a cell viability assay. Biotechniques, 34: 260-264.

Metz, S.W., Feenstra, F., Villoing, S., van 
Hulten, M.C., van Lent, J.W., Koumans, J., Vlak, J.M., Pijlman, G.P. 2011. Low Temperature-Dependent Salmonid Alphavirus Glycoprotein Processing and Recombinant Virus-Like Particle Formation. PLoS ONE, 6: e25816.

Mulvania, T., Hayes, B., Hedin, D.A. 2004. Flow cytometric assay for rapid, accurate determination of baculovirus titers. Bioprocess J., 3:47-53.

O Reilly, D.R, Miller, L.K., Luckow, V.A. 1992. Baculovirus Expression Vector: A Laboratory Manual, W.H. Freeman, NY.

Pouliquen, Y., Kolbinger, F., Geisse, S., Mahnke, M. 2006. Automated baculovirus titration assay based on viable cell growth monitoring using a colorimetric indicator, Biotechniques, 40:282-292.

Qi, J., Liu, T., Pan, J., Miao, P., Zhang, C. 2015. Rapid baculovirus titration assay based on viable cell side scatter (SSC). Anal. Chim. Acta, 879: 58-62.

Reed, L.J., Muench, H.1938. A simple method of estimating fifty per cent endpoints. Am. J. Epidemiol., 27: 493-
497.

Roberts, L.O, Seamons, R.A, Belsham, G.J. 1998. Recognition of picornavirus internal ribosome entry sites within cells; influence of cellular and viral proteins. RNA, 4: 520-529.

Roldao, A., Carrondo, M.J.T., Alves, P.M., Oliveira, R.2008. Stochastic simulation of protein expression in the baculovirus/insect cells system. Comp. Chem. Eng., 32: 68-77.

Shen, C.F., Meghrous, J., Kamen, A. 2002. Quantitation of baculovirus particles by flow cytometry. J. Virol. Methods, 105:321-330.

Trichas, G., Begbie, J., Srinivas, S. 2008. Use of the viral $2 \mathrm{~A}$ peptide for bicistronic expression in transgenic mice. $B M C$ Biol., 6:40.

Wang, W., Cheng, T., Ma, K., Xia, D.Z., Wang, Y.M., Liu, J., Du, H.L., Shih, J.W.K., Zhang, J., Zhao, Q.J., Xia, N.S. 2013. Development of a novel baculovirus titration method using the enzyme-linked immunosorbent spot (ELISPOT) assay. J. Virol. Methods, 188:114-120.

\section{How to cite this article:}

Deepika Bisht, Supriya Bhattacharya, Lekshmi S. Rajan, Mashidur Rana, Ram Bachan, T. Rama, Mohini Saini and Praveen K. Gupta. 2018. A Rapid Method for Titration of Recombinant Baculovirus Stock Based on Bicistronic Expression of Green Fluorescent Protein (GFP) Reporter. Int.J.Curr.Microbiol.App.Sci. 7(12): 1141-1149.

doi: https://doi.org/10.20546/ijcmas.2018.712.140 